\title{
Phase 3 International Trial of Adjuvant Whole Brain Radiotherapy (WBRT) or Observation (OBS) Following Local Treatment of 1-3 Melanoma Brain Metastases (MBMs)
}

Fogarty, GB; Dolven-Jacobsen, K ; Morton, RL; Hruby, G; Nowak, AK; Vardy, JL Drummond, KI'; Dhillon, HM; Mandel, C; Scolyer, RA' ; Shivalingam, B; Middleton, MR; Burmeister, B; Roos, DE; Lo, SN; Reisse, $\mathrm{CH}$; Paton, EI; Williams, NC; Thompson, JF; Hong, A

\section{Purpose/Objective(s)}

The role of adjuvant WBRT in MBMs is controversial. This phase 3 trial compares WBRT with Obs following local treatment of 1-3 MBMs.

\section{Materials/Methods}

The primary endpoint is distant intracranial failure (DIF) within 12 months of randomization. The a priori neurocognitive function (NCF) endpoint is Hopkins Verbal Learning Test-Revised (HVLT-R) delayed recall at 4 months. Secondary endpoints include local failure (LF), overall survival (OS) and global quality of life (QoL). Analyses were conducted on intention-to-treat basis with nominal two-sided significance level 5\%. Drug therapy was allowed. Effective drugs became available during the trial and their impact was analyzed.

\section{Results}

Of 586 eligible patients, 215 consented from 31 sites in 3 countries (Australia, UK and Norway) between 2009 and 2017. Eight (0.04\%) who withdrew or had no data collected were excluded. 107 were randomized to Obs and 100 to WBRT. Mean age was 62 years, $67 \%$ were males, $61 \%$ with single MBM of mean size $2 \mathrm{~cm}, 67 \%$ had extracranial disease at randomization. The two arms were well matched. NCF was completed by English speakers; 50 WBRT and 70 Obs pts at baseline, declining to 26 and 
35 respectively at 4 months. Within 12 months, 54 (50.5\%) Obs patients had DIF compared with 42 (42.0\%) WBRT patients (OR 0.71; 95\% CI 0.411.23; $\mathrm{p}=0.222)$. There was no difference in $\mathrm{LF}(\mathrm{p}=0.100)$ or OS (log-rank $\mathrm{p}=0.861$ ). 53\% (Obs) and 59\% (WBRT) patients were alive at 12 months. There was no significant between-group difference in mean intervention effect on global QoL ( $\mathrm{p}=\mathrm{0.083}$ ). Patients who received T-cell checkpoint inhibitors and/or mitogen-activated protein kinases (MAPK) pathway inhibitors and WBRT before or within 12 months of randomization had DIF rate $29 \%$ compared with Obs and no systemic therapy had $44 \%$, but this was not significant $(\mathrm{p}=0.228)$. Obs patients had greater relative improvement from baseline in HVLT-R at every timepoint. At 4 months, Obs had 20.9\% improvement from baseline in HVLT-R-delayed recall compared to $2.7 \%$ decline in WBRT; overall adjusted average intervention effect 23.6\% (95\%CI 9.0, 38.2; $\mathrm{p}=0.0018$ ). There was no difference in time to cognitive failure or in proportions with global cognitive impairment.

\section{Conclusion}

This level one evidence shows WBRT does not improve outcomes in MBMs. This practice-changing trial justifies the recent move away from WBRT that occurred during the course of the trial. 